\title{
New insights into the regulation of the Saccharomyces cerevisiae UGA4 gene: two parallel pathways participate in carbon-regulated transcription
}

Correspondence

Susana Correa García correa@qb.fcen.uba.ar

Received 31 May 2007

Revised 3 July 2007

Accepted 16 July 2007

\author{
Carlos Luzzani, Sabrina Beatriz Cardillo, Mariana Bermúdez Moretti† \\ and Susana Correa García†
}

Departamento de Química Biológica, Facultad de Ciencias Exactas y Naturales, Universidad de Buenos Aires, Ciudad Universitaria, Pabellón II, Piso 2. C1428EGA, Buenos Aires, Argentina

\begin{abstract}
The Saccharomyces cerevisiae UGA4 gene, which encodes the $\gamma$-aminobutyric acid (GABA) and $\delta$-aminolaevulinic acid (ALA) permease, is well known to be regulated by the nitrogen source. Its expression levels are low in the presence of a rich nitrogen source but are higher when a poor nitrogen source is used. In addition, GABA can induce UGA4 expression when cells are grown with proline but not when they are grown with ammonium. Although vast amounts of evidence have been gathered about UGA4 regulation by nitrogen, little is known about its regulation by the carbon source. Using glucose and acetate as rich and poor carbon source respectively, this work aimed to shed light on hitherto unclear aspects of the regulation of this gene. In poor nitrogen conditions, cells grown with acetate were found to have higher UGA4 basal expression levels than those grown with glucose, and did not show UGA4 induction in response to GABA. Analysis of the expression and subcellular localization of the transcription factors that regulate UGA4 as well as partial deletions and site-directed mutations of the UGA4 promoter region suggested that there are two parallel pathways that act in regulating this gene by the carbon source. Furthermore, the results demonstrate the existence of a new factor operating in UGA4 regulation.
\end{abstract}

\section{INTRODUCTION}

The yeast Saccharomyces cerevisiae is widely used as a model organism to study the function of mammalian proteins. The GATA family of DNA-binding proteins, responsible for regulating globin gene expression (Weiss \& Orkin, 1995) and a diverse set of developmental functions in animal cells (Kelley et al., 1993; Laverriere et al., 1994), is one of the gene families shared by S. cerevisiae and higher eukaryotes. Yeast GATA proteins Gln3, Gat1/Nill, Dal80/ Uga43 and Gzf3/Deh1 are the main regulators of nitrogen catabolism gene expression that are responsible for the phenomenon known as nitrogen catabolite repression (NCR). The expression of the UGA4 gene, which encodes the $\gamma$-aminobutyric acid (GABA) and $\delta$-aminolaevulinic acid (ALA) permease in S. cerevisiae, depends on GABA induction and NCR (Andre et al., 1993; Bermudez Moretti et al., 1996). Induction of this permease requires at least two positive-acting proteins, the specific Uga3p factor and the pleiotropic Dal81p/Uga35p factor (Andre et al., 1995;

Abbreviations: ALA, $\delta$-aminolaevulinic acid; GABA, $\gamma$-aminobutyric acid; NCR, nitrogen catabolite repression; UAS, upstream activating sequence.

tMembers of the Research Career Programme of CONICET.
Bricmont et al., 1991). These factors act through a $19 \mathrm{bp}$ CG-rich upstream activating sequence, $\mathrm{UAS}_{\mathrm{GABA}}$. The promoter region of $U G A 4$ also contains four adjacent repeats of the heptanucleotide $5^{\prime}$-CGAT(A/T)AG-3', which constitute a $\mathrm{UAS}_{\mathrm{GATA}}$ element. This element can potentially confer high levels of expression in the absence of inducer. Nevertheless, the basal expression levels of the UGA4 gene in uninduced cells grown with a poor nitrogen source such as proline are low since there is a strong repression mechanism involving Dal80p/Uga43p, a pleiotropic regulatory factor (Andre et al., 1995; Bricmont et al., 1991; Cunningham et al., 1994). Gln3p, another GATA transcription factor, upregulates the expression of UGA4 in the presence of inducer by competing with Dal80p/Uga43p for binding to the $\mathrm{UAS}_{\mathrm{GATA}}$ sequence (Coffman et al., 1997; Soussi-Boudekou et al., 1997). The outcome of this competition influences basal levels of transcription. The role of the other positive and negative GATA factors, Gat1 and Gzf3 respectively, in UGA4 regulation has been poorly studied.

NCR is superimposed on the above regulation: in cells grown with a rich nitrogen source such as ammonium or glutamine, Ure2p, a pre-prionic cytoplasmic protein, prevents nuclear localization of Gln3p by retaining it in 
the cytoplasm, consequently preventing its activity (Kulkarni et al., 2001).

Gat1 was thought to act in a way similar to Gln3, but whereas a Gln3-Ure2 complex has been isolated from cells in which NCR-sensitive transcription is repressed, a similar Gat1Ure2 complex has not yet been reported (Tate et al., 2006).

Many reports have been published assessing the relationship between nuclear localization and phosphorylation levels of Gln3; emerging results show a lack of correlation between these parameters. However, Gln3 intracellular localization and NCR do correlate well (Tate et al., 2005). It must be noted that much less is known about Gat1 phosphorylation and localization in response to nutrient availability (Kulkarni et al., 2006).

TOR1/2 are phosphatidylinositol kinase-related proteins, which are inhibited by rapamycin and sense nutrients, specifically carbon and nitrogen quality, regulating gene expression. These kinases are, at least in part, involved in Gln3 and Gat1 phosphorylation (Bertram et al., 2002). It has been reported that the activation of Gln 3 and Gat1 is not identical, that they control distinct (although overlapping) sets of genes and that low-quality carbon or nitrogen activates Gln3 or Gat1 differentially (Crespo et al., 2002; Kuruvilla et al., 2001; Shamji et al., 2000). Moreover, the phosphorylation state of Ure2, the anchor protein of the positive GATA factors in the cytoplasm when carbon and nitrogen nutrients are abundant (Beck \& Hall, 1999), responds not to the nitrogen availability but rather to the carbon source (Kuruvilla et al., 2002).

Evidence indicates that not only nitrogen but also carbon nutrient quality controls the expression of some NCR genes (Shamji et al., 2000) and that Gln3 and Gat1 localization and/or phosphorylation depend on the quality of the available carbon and/or nitrogen source (Bertram et al., 2002; Crespo et al., 2002; Kulkarni et al., 2006; Tate et al., 2005).

Since the UGA4 gene is tightly regulated and some of the transcription factors participating in its regulation (i.e. Gln3 and Gat1) have been linked to NCR gene expression in response to the quality of the carbon source, the aim of our work was to get further insights into the regulation of this gene by the carbon source. We analysed the expression of UGA4 and the role of the GATA factors and the target DNA sequences on the UGA4 promoter involved in the regulation of the UGA4 gene.

\section{METHODS}

Strains and media. The Saccharomyces cerevisiae strains used in this study, isogenic to the wild-type $\Sigma 1278$ b, were 23344c (MAT $\alpha$ ura3),

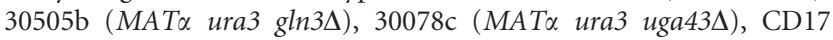

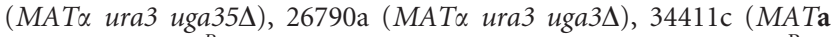
ura3 nil1 ${ }^{\circ}\left(\operatorname{Kan}^{R}\right)$ gln3 $\left.{ }^{\circ}\right)$ and $32164 \mathrm{~b}\left(\operatorname{MAT\alpha }\right.$ ura3 nill $\left.{ }^{\circ}\left(\operatorname{Kan}^{\mathrm{R}}\right)\right)$. These strains were kindly provided by Professor S. Vissers (Université Libre de Bruxelles, Belgium). Cells were grown in minimal medium containing $0.17 \%$ Difco yeast nitrogen base (YNB without amino acids and ammonium sulfate) with $2 \%$ glucose or $2 \%$ potassium acetate as carbon source, and $10 \mathrm{mM}$ proline or $10 \mathrm{mM}$ ammonium sulfate as nitrogen source. Yeast strains were transformed using the methodology described by Gietz \& Woods (2002).

Construction of fusion plasmids. All procedures for manipulating DNA were standard ones (Sambrook et al., 1997). 5'-Regulatory regions and part of the coding regions of the UGA4 $(-583$ to +15 , with respect to the ATG initiation codon), UGA43 $(-848$ to +48$)$, GZF3 $(-790$ to +21$)$, GLN3 $(-826$ to +44$)$, GAT1 $(-787$ to +48$)$, UGA35 $(-393$ to +51$)$ and UGA3 $(-261$ to +24$)$ genes were fused in-frame to the lac $Z$ gene lacking its first seven codons, in the plasmid YEP357 (Myers et al., 1986). This plasmid carries the URA3 selectable marker complementing the uracil auxotrophy of the yeast strains used. DNA fragments were generated by PCR amplification using $\Sigma 1278$ b genomic DNA as template. Primers used in these constructions are listed in Table 1. All fusion plasmids were verified by DNA sequence analysis. Escherichia coli DH5 $\alpha$ was used to amplify and maintain the plasmids.

Two nested $5^{\prime}$ deletions of UGA4 called $\mathrm{UAS}_{\mathrm{GATA}} \Delta$ (i.e. without the $\mathrm{UAS}_{\mathrm{GATA}}$ sequence) and UAS $\Delta$ (i.e. without the $\mathrm{UAS}_{\mathrm{GATA}}$ and $\mathrm{UAS}_{\mathrm{GABA}}$ sequences) (see Table 1 and Fig. 5) fused to the lac $Z$ reporter gene were also generated using the strategy described above.

In vitro site-specific mutagenesis was carried out according to Strachan \& Read (1999). Briefly, two PCRs, using primers mut1 and F-UGA4 or primers mut2 and R-UGA4 and the fusion plasmid carrying UGA4-lacZ as template, were performed. Primers mutl and mut2 (Table 1) contain the specific pre-determined mutation (Fig. 5a), located in a central segment. After the two products were combined, denatured and allowed to reanneal, the DNA polymerase extended the $3^{\prime}$ end of heteroduplexes with recessed $3^{\prime}$ ends. Thereafter, a full-length product ( $\left.\mathrm{UAS}_{\mathrm{GABA}} \mathrm{mut}\right)$ with the introduced mutation in a central segment was amplified by PCR using the outer primers F-UGA4 and R-UGA4 and then it was fused to the lacZ gene in YEP357.

$\boldsymbol{\beta}$-Galactosidase assays. Cells were grown on the indicated media to $\mathrm{OD}_{570} 0.5-0.9$, then harvested and transferred to the same fresh medium with or without $0.1 \mathrm{mM}$ GABA. An aliquot $(10 \mathrm{ml})$ of each culture was collected by centrifugation after $2 \mathrm{~h}$ incubation at $30{ }^{\circ} \mathrm{C}$ and resuspended in $2 \mathrm{ml}$ buffer $Z$ (Miller, 1972). $\beta$-Galactosidase activity measured according to Miller (1972) was expressed as Miller units. Results are shown as mean \pm standard deviation of duplicates within a representative assay. At least duplicate assays for each of two independent transformants were performed. The deviation of these values from the mean was less than $15 \%$.

Fluorescence microscopy. 23344c cells transformed with the plasmid pRR482 (kindly provided by Dr T. Cooper, University of Tennessee, Memphis, USA), containing the full-length coding sequence of Gln3 fused to GFP under the GAL1 promoter, were grown on a plate of ammonium-glucose medium. Cells were picked from the plate, transferred to fresh liquid medium containing $4 \%$ galactose and $10 \mathrm{mM}$ proline as the nitrogen source and incubated for $30 \mathrm{~min}$. Then cells were collected, washed and transferred again to the indicated fresh medium. After a 90 min incubation, cells from a $5 \mathrm{ml}$ culture were fixed with $70 \%$ ethanol in $50 \mathrm{mM}$ Tris $/ \mathrm{HCl}$ $\mathrm{pH}$ 7.5. After $1 \mathrm{~h}$ of incubation, an overnight RNase treatment was performed and nuclei were stained with a $50 \mu \mathrm{g} \mathrm{ml}^{-1}$ propidium iodide solution in PBS. Analysis was carried out by using a laserscanning confocal microscope (Fluoview FV300 BS61; Olympus). Images were acquired simultaneously into acquisition channels with the FLUOVIEW FV300 (version 3.3) acquisition/analyser program. Photographs were imported to Adobe Photoshop 8.0 for image management. 
Table 1. Oligonucleotide primers

Bold type letters represent the restriction sites.

\begin{tabular}{|c|c|}
\hline Primer & Sequence $\left(5^{\prime}-3^{\prime}\right)$ \\
\hline F-UGA4 & CGCGGAATTCGACAATTTCTTCAATCATTGAAATG \\
\hline R-UGA4 & CCСCAAGCTTCATACTCATTGTTAGTAATAATAAATTATAAGACCT \\
\hline F-UGA43 & CGCGGAATTCTTGTTGTAGAGCCGGTGCAT \\
\hline R-UGA43 & CCCCAAGCTTAGCTGAAAGTGTAGGCGA \\
\hline F-GZF3 & CCGGAATTCCTGTATCCTTGGTC \\
\hline R-GZF3 & CGCGGATCCAGTTGTAGCCTG \\
\hline F-GLN3 & CGCGGAATTCCTGATCGAGATCCTCC \\
\hline R-GLN3 & CCCCAAGCTTCAGCAGGTCGTACAGCT \\
\hline F-GAT1 & CCGGAATTCAGCATTTTTCACG \\
\hline R-GAT1 & CCCAAGCTTGAACAGAACAGGG \\
\hline F-UGA35 & CGCGGAATTCGAGCTCCCACAGTGATGTAG \\
\hline R-UGA35 & CCCCAAGCTTGCTCTTCGTAGGCGATGC \\
\hline F-UGA3 & CGCGGAATTCACGTTTTGCGGGCATCACGT \\
\hline R-UGA3 & CCCCAAGCTTCAGCTTCTCCACGCCATAATT \\
\hline F-UAS $\mathrm{GATA}_{\mathrm{T}} \Delta$ & CGCGGAATTCCCAAAAACCGCCGGCGGCAATTT \\
\hline F-UAS $\Delta$ & CGCGGAATTCTTCGAGATGTTTTATGTCATGTTGG \\
\hline mut1 & ACATAAAACATCTCGAAATTATTACTAATGGTTTTTGGCGCACGA \\
\hline mut2 & TCGTGCGCCAAAAACCATTAGTAATAATTTCGAGATGTTTTATGT \\
\hline
\end{tabular}

\section{RESULTS}

\section{Nitrogen and carbon sources regulate UGA4 gene expression}

$\beta$-Galactosidase activity was measured in wild-type cells (strain $23344 \mathrm{c}$ ) transformed with a plasmid carrying the $5^{\prime}$ regulatory region and part of the coding region of UGA4 fused in-frame to lacZ (YEP357 UGA4:: lacZ) grown with different nitrogen and carbon sources. Both basal and GABA-induced UGA4 expression were analysed. When glucose, a glycolytic substrate, was used in the presence of the poor nitrogen source proline, the basal expression level was low and significant GABA induction was detected, whereas in the presence of ammonium, a rich nitrogen source, both basal and GABA-induced levels were repressed (Fig. 1). These were the expected results since UGA4 is an inducible gene and it is subject to NCR (Talibi et al., 1995). When the carbon source was acetate, a gluconeogenic substrate, basal expression levels in cells grown in derepressed conditions (i.e. proline as nitrogen source) were higher than those observed in cells from glucoseproline medium and there was no significant GABA induction. NCR was still observed when acetate was the carbon source. These results show that UGA4 is not only regulated by the nitrogen source, as has been extensively described, but also by the carbon source.

\section{Effect of transcription factors acting through the UAS $S_{\text {GATA }}$ element in the UGA4 promoter}

We decided to study if the molecular mechanisms by which UGA4 expression is regulated by carbon source involve the GATA factors.
Firstly, the expression of the four GATA factors in wildtype cells grown with either glucose or acetate was determined using $\beta$-galactosidase assays. These experiments were performed using proline as the nitrogen source since expression values obtained with ammonium were too low to detect any differences (data not shown). A high expression of UGA43 and a low expression of GZF3 were

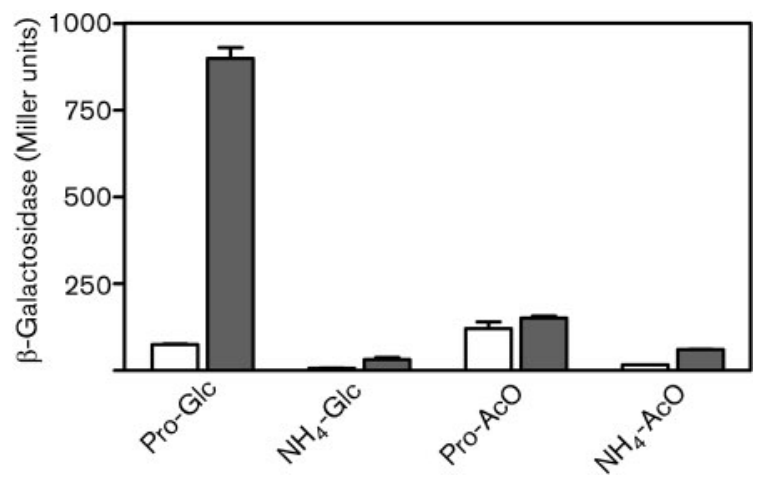

Fig. 1. Effect of different carbon and nitrogen sources on UGA4 expression. $\beta$-Galactosidase activity was determined in extracts from wild-type cells (strain 23344c) carrying the UGA4:: lacZ fusion gene. Cells grown on minimal medium containing proline and glucose (Pro-Glc), ammonium and glucose $\left(\mathrm{NH}_{4}-\mathrm{Glc}\right)$, proline and acetate (Pro-AcO) or ammonium and acetate $\left(\mathrm{NH}_{4}-\mathrm{AcO}\right)$ as nitrogen and carbon sources were collected and transferred to the same fresh media containing (filled bars) or not (open bars) $0.1 \mathrm{mM}$ GABA. After a $2 \mathrm{~h}$ incubation samples were taken and $\beta$ galactosidase activity was measured. Results are shown as mean $\pm S D$ of duplicates within a representative assay. 
Table 2. Effect of different carbon sources on expression of GATA factors

$\beta$-Galactosidase activity was determined in extracts from wild-type cells (strain 23344c) carrying the UGA43::lacZ, GZF3::lacZ, GLN3: : lacZ or GAT1 : : lacZ fusion. Cells grown in minimal medium containing proline as the nitrogen source and glucose or acetate as the carbon source were transferred to the same fresh media, and after a $2 \mathrm{~h}$ incubation samples were taken and $\beta$-galactosidase activity was measured. Results are shown as mean $\pm \mathrm{SD}$ of duplicates within a representative assay.

\begin{tabular}{|lcccc|}
\hline \multirow{2}{*}{$\begin{array}{l}\text { Carbon } \\
\text { source }\end{array}$} & \multicolumn{4}{c}{$\boldsymbol{\beta}$-Galactosidase activity (Miller units) } \\
\cline { 2 - 5 } & UGA43 & GZF3 & GLN3 & GAT1 \\
\hline Glucose & $680.5 \pm 27.5$ & $85.7 \pm 23.5$ & $223.9 \pm 33.2$ & $8.0 \pm 1.8$ \\
Acetate & $538.9 \pm 66.4$ & $79.1 \pm 26.4$ & $29.7 \pm 4.7$ & $2.7 \pm 0.8$ \\
\hline
\end{tabular}

observed and they did not vary with the carbon source (Table 2). Thus, changes in expression of the negative GATA factors do not explain the observed variation in UGA4 expression.

The expression level of the positive GATA factor GAT1 was very low in both growth conditions tested. The expression of the other positive GATA factor, GLN3, in the presence of glucose was significantly higher than when acetate was used. Thus, there is a correlation between GABA induction of UGA4 gene (Fig. 1) and GLN3 expression (compare Fig. 1 and Table 2).

It has been extensively described that UGA4 expression is, at least in part, the result of the competition between positive and negative GATA factors for the binding to the $\mathrm{UAS}_{\mathrm{GATA}}$ element (Andre et al., 1995; Cunningham et al., 1994). Gat1 and Gzf3 regulate UGA4 expression in the presence of a preferred nitrogen source (Coffman et al., 1997; Soussi-Boudekou et al., 1997) while Gln3 and Uga43 are the main GATA factors acting on the $\mathrm{UAS}_{\mathrm{GATA}}$ element of the UGA4 promoter in our experimental conditions, i.e. proline-glucose medium. To determine if the absence of GABA induction of $U G A 4$ gene in cells grown on acetate is
Table 3. Effect of the repressor Uga43 on UGA4 regulation by carbon source

$\beta$-Galactosidase activity was determined in extracts from wild-type (strain 23344c) and uga43A (strain 30078c) cells carrying the UGA4: lacZ fusion. Cells grown on minimal medium containing proline and glucose or acetate were collected and transferred to the same fresh media containing or not $0.1 \mathrm{mM}$ GABA. After a $2 \mathrm{~h}$ incubation samples were taken and $\beta$-galactosidase activity was measured. Results are shown as mean \pm SD of duplicates within a representative assay.

\begin{tabular}{|llcc|}
\hline \multirow{2}{*}{ Genotype } & Carbon & \multicolumn{2}{c|}{$\boldsymbol{\beta}$-Galactosidase activity (Miller units) } \\
\cline { 3 - 4 } & source & -GABA & +GABA \\
\cline { 3 - 4 } & & $75.1 \pm 2.8$ & $898.9 \pm 44.4$ \\
Wild-type & Glucose & $133.0 \pm 26.3$ & $151.4 \pm 11.0$ \\
& Acetate & $1363.8 \pm 154.9$ & $1576.2 \pm 410.3$ \\
uga43 $\Delta$ & Glucose & $185 \pm 25.5$ & $131.3 \pm 28.5$ \\
& Acetate & \\
\hline
\end{tabular}

due to a strong binding of the repressor Uga43 to $\mathrm{UAS}_{\mathrm{GATA}}, \mathrm{UGA4}$ expression was measured in cells deficient in this negative factor. As expected, UGA4 levels were high in uga $43 \Delta$ cells grown on glucose even in the absence of GABA; however, in uga $43 \Delta$ cells grown on acetate, values were similar to those obtained in wild-type cells (Table 3 ). These data clearly show that the effect of carbon source on UGA4 does not depend on Uga43.

One reason for the low expression of UGA4 in acetategrown cells could be that GLN3 levels, even in the absence of Uga43, are not enough to allow UGA4 GABA induction. On the other hand, it is well known that the activity of the positive GATA factors depends on their intracellular localization; thus, another reason for UGA4 expression profiles in acetate could be a cytoplasmic accumulation of Gln3. Therefore, subcellular localization of the fusion protein Gln3-GFP was determined by fluorescence microscopy of cells grown with different carbon sources (Fig. 2). As expected, in wild-type cells grown on glucose, Gln3 was mainly observed in the nucleus. In acetate-grown cells, GFP

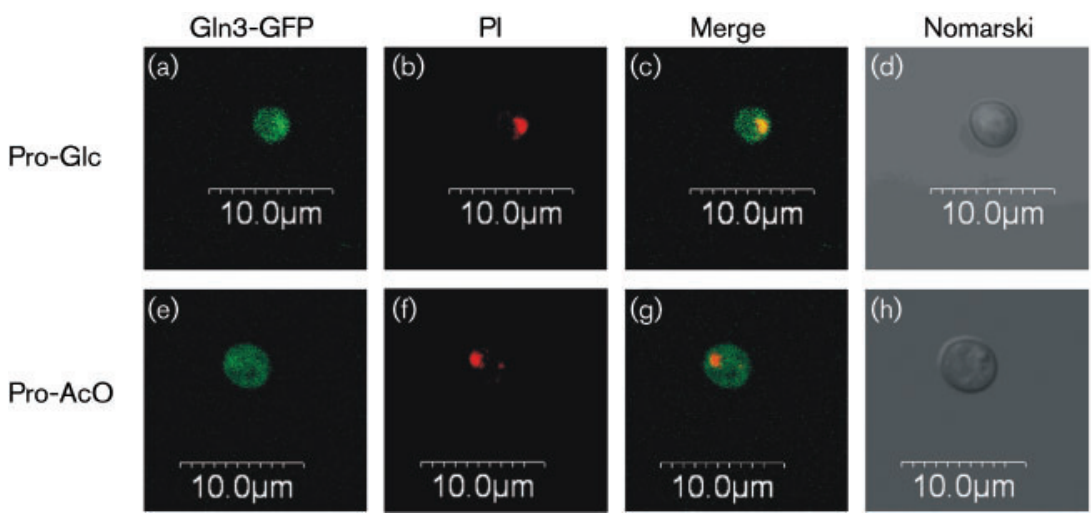

Fig. 2. Gln3 localization in cells grown on different carbon sources. Subcellular localization of Gln3-GFP was determined by confocal microscopy in the wild-type strain (23344c) transformed with pRR482. After $30 \mathrm{~min}$ of $4 \%$ galactose induction, cells were transferred and incubated for 90 min in different minimal media containing proline and glucose $(a-d)$ or proline and acetate $(e-h)$. Nuclear staining was performed with propidium iodide $(\mathrm{PI})$ as described in Methods. Representative images are shown. 
fluorescence was preferentially seen in the nucleus, although some fluorescence was detected in the cytoplasm. In cells incubated in glucose or acetate the protein Gln3GFP was overexpressed. As expression of this fusion protein is driven by GAL1, when cells were transferred to glucose the shut-off of the promoter was probably much more drastic than in acetate. In this case, the amount of Gln3-GFP in acetate-grown cells would be higher than in glucose-grown cells. In any case, since the fluorescence was mainly observed in the nucleus, the absence of induction of UGA4 gene in acetate medium can not be attributed to the absence of Gln3 in the nucleus. Although the Gln3 localization patterns were slightly different, the observation of a large number of cells from independent experiments, where several Gln3-GFP expression levels were assayed, led us to the belief that carbon source may regulate $G \ln 3$ intracellular localization.

To further analyse the role of the GATA factors in carbon regulation, UGA4 expression was measured in mutant strains deficient in the GLN3 and/or GAT1 genes. UGA4 expression was almost undetectable in a $g \ln 3 \Delta$ strain irrespective of the carbon source, whereas the deletion of GAT1 did not seem to affect UGA4 expression (Fig. 3a, b). Interestingly, in the double mutant $g \ln 3 \Delta$ gat $1 \Delta$ the expression levels of UGA4 were similar to those observed in wild-type cells. This apparent discrepancy was explained by analysing the expression of each negative GATA factor in these strains (Fig. 4a-d). The data show that in $g \ln 3 \Delta$ cells there was significant expression of the negative factor $U G A 43$ while in $g \ln 3 \Delta$ gat $1 \Delta$ cells almost no expression of both negative factors was observed, allowing the recovery of UGA4 expression levels. In other words, the absence of the two activators is compensated by the simultaneous absence of the two repressors. It must be noted that in the double mutant strain $(g \ln 3 \Delta$ gat $1 \Delta)$, when both positive GATA factors were absent and the expression of the negative ones was negligible, the expression profiles of UGA4 were significantly different depending on the carbon source: UGA4 levels were lower, and no GABA induction was observed, in cells from acetate compared to those from glucose (Fig. 3).

In conclusion, although the expression levels and probably the intracellular localization of Gln3 are modulated by the carbon source, indicating that this factor may be involved in carbon regulation of UGA4, our results suggest that this regulation is also mediated by other factors.

\section{Effect of transcription factors acting through the UAS $_{\mathrm{GABA}}$ element in the UGA4 promoter}

The transcription factors Uga3 and Uga35 regulate UGA4 expression, acting through the $\mathrm{UAS}_{\mathrm{GABA}}$ element. Thus, the expression of these factors in wild-type cells was analysed in order to establish their involvement in UGA4 regulation by carbon source. UGA35 levels did not vary and were very low on both carbon sources (less than 25 Miller units) whereas UGA3 levels in glucose medium were three times higher than those measured in acetate medium (160 Miller units in glucose and 53 Miller units in acetate).

Results of UGA4 expression in strains deficient in UGA35 and $U G A 3$ were not reproducible although always low. It must be noted that these strains do not grow well in minimal media. Thus, the involvement of Uga3 in the effect of the carbon source on UGA4 gene (i.e. GABA induction in glucose but not in acetate) could not be determined by this methodology.

\section{Role of the UAS elements in the response of UGA4 to carbon source}

In order to establish the DNA sequences participating in carbon regulation of $U G A 4$, modifications in the promoter
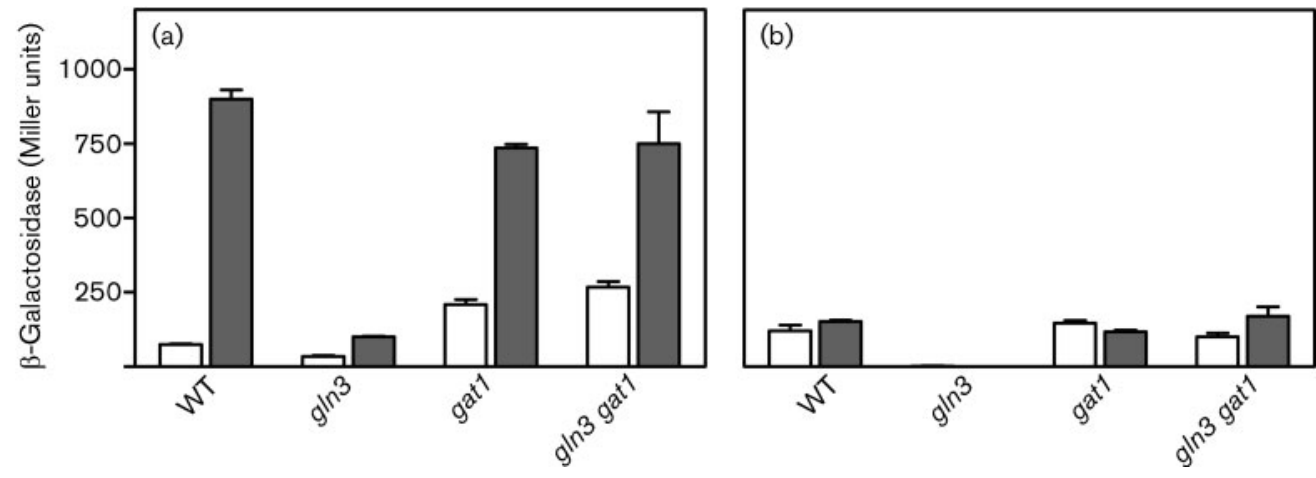

Fig. 3. Effect of different carbon sources on UGA4 expression in cells deficient in the GATA factors GIn3 and Gat1. $\beta$ -

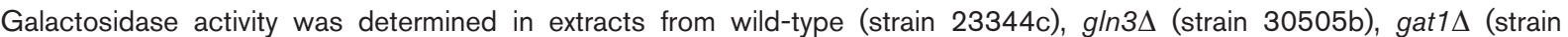

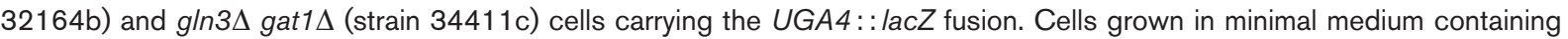
proline as the nitrogen source and glucose (a) or acetate (b) as the carbon source were collected and transferred to the same fresh media containing (filled bars) or not (open bars) $0.1 \mathrm{mM}$ GABA. After a $2 \mathrm{~h}$ incubation, samples were taken and $\beta$ galactosidase activity was measured. Results are shown as mean \pm SD of duplicates within a representative assay. 

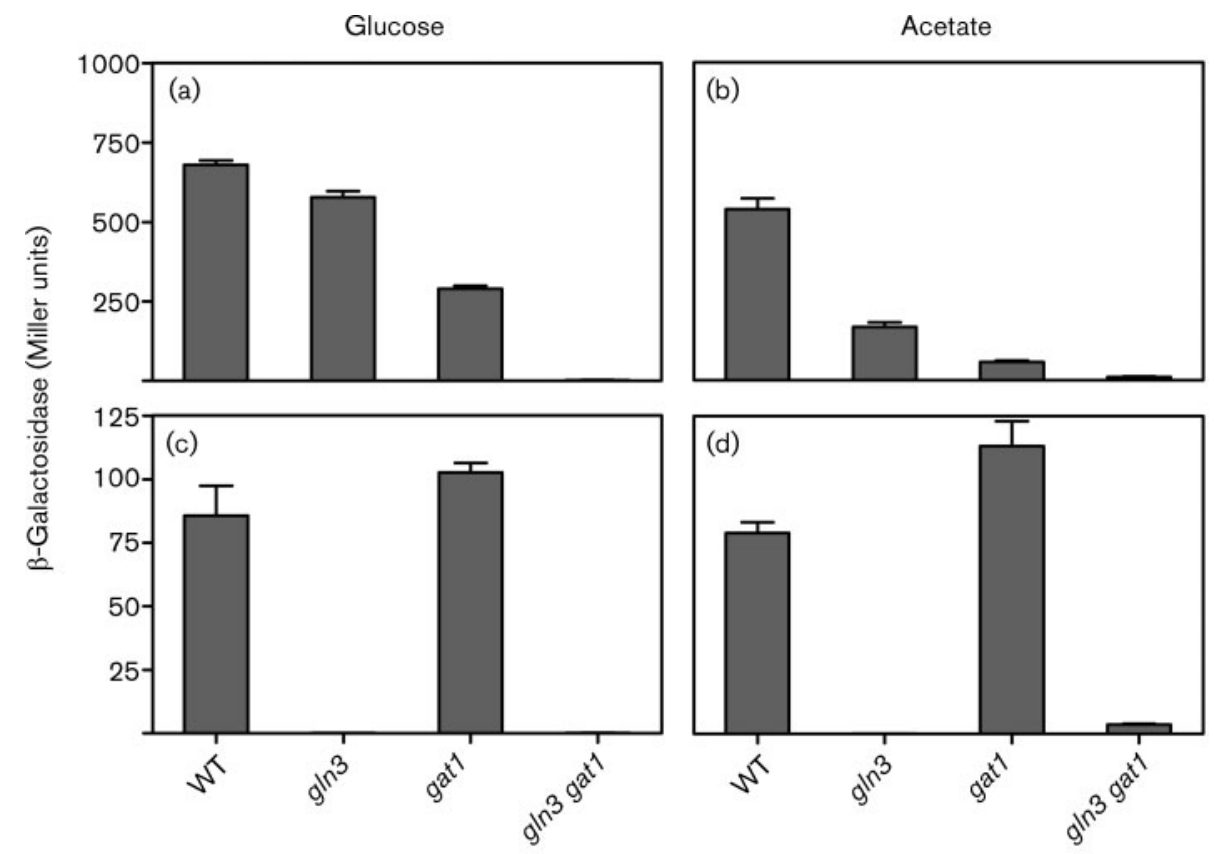

Fig. 4. Effect of different carbon sources on UGA43 and GZF3 expression in cells deficient in the GATA factors Gln 3 and Gat1. $\beta$-Galactosidase activity was determined in extracts from wild-type (strain 23344c), gln3 $\Delta$ (strain 30505b), gat1 $\Delta$ (strain

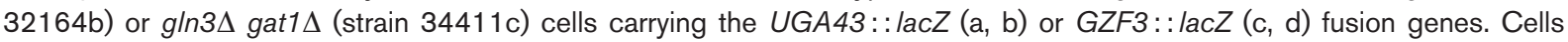
grown in minimal medium containing proline as the nitrogen source and glucose $(a, c)$ or acetate $(b, d)$ as the carbon source were collected were transferred to the same fresh media, and after a $2 \mathrm{~h}$ incubation, samples were taken and $\beta$-galactosidase activity was measured. Results are shown as mean \pm SD of duplicates within a representative assay. The deviation of these values from the mean was less than $15 \%$.

were introduced and cloned upstream of lacZ. Expression driven by a $5^{\prime}$ deletion of the UGA4 promoter lacking the $\mathrm{UAS}_{\text {GATA }}$ element in wild-type cells was lower than when the whole promoter was used but the effect of the carbon source remained unchanged (Fig. 5a, b, c). These results indicate that other elements besides GATA participate in this regulation. No differences between wild-type cells grown with glucose or acetate were observed in lac $Z$ expression driven by a $5^{\prime}$ deletion of the UGA4 promoter lacking both $\mathrm{UAS}_{\mathrm{GATA}}$ and $\mathrm{UAS}_{\mathrm{GABA}}$ elements (Fig. 5a, b, d). Taken together these results indicate that the UGA4 DNA sequence -406 to -385 is involved in carbon regulation. Expression levels driven by $\mathrm{UAS}_{\mathrm{GABA}}$ mut were high with both glucose and acetate, indicating that this element is responsible for carbon regulation (Fig. 5a, b, e). Moreover, a repressor seems to be acting on the native $\mathrm{UAS}_{\mathrm{GABA}}$ element (compare Fig. 5b, e).

\section{DISCUSSION}

Data presented in this work provide clear evidence that the quality of the carbon source regulates UGA4 expression and that this regulation is the result of at least two parallel pathways. Different elements present in the $5^{\prime}$ regulatory region of UGA4 are involved in this regulation. UGA4 expression is regulated by carbon source through Gln3 acting on the $\mathrm{UAS}_{\mathrm{GATA}}$ element and through another factor, as yet unknown, acting on the $\mathrm{UAS}_{\mathrm{GABA}}$ element.

Kuruvilla et al. (2002), dissecting glucose signalling in yeast, found a set of genes regulated by carbon source but UGA4 was not one of them. Moreover, this research group postulated that carbon and nitrogen quality signal preferentially through the multiprocessing Tor proteins to Gat1 and Gln3, respectively (Kuruvilla et al., 2001). Our results do not agree with these findings since the activity of Gat1 on UGA4 does not seem to depend on the carbon source, wheras Gln3 activity is clearly regulated by carbon quality.

We found that in the presence of the rich carbon source glucose UGA4 expression is low and a significant increase of this expression is observed when the inducer GABA is added. In contrast, UGA4 expression in cells grown on the non-fermentable carbon source acetate is higher than that observed in glucose-grown cells and it is not dependent on the presence of GABA. We conclude that the expression of the GATA factor Gln3 is also modulated by the carbon source. Moreover, the slight changes in the subcellular localization patterns observed suggest that Gln3 activity could be also regulated by carbon source at this level. Thus, UGA4 expression must be affected by the different patterns 
(a)

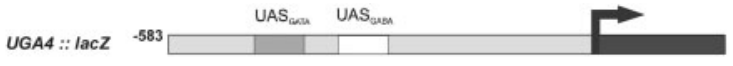

AAAAACCGCCGGCGGCAAT
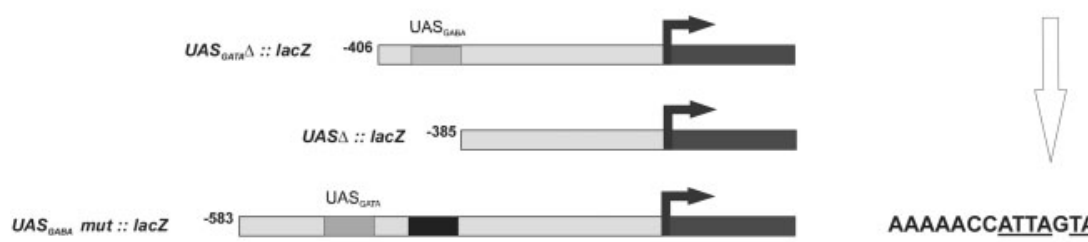

UAS

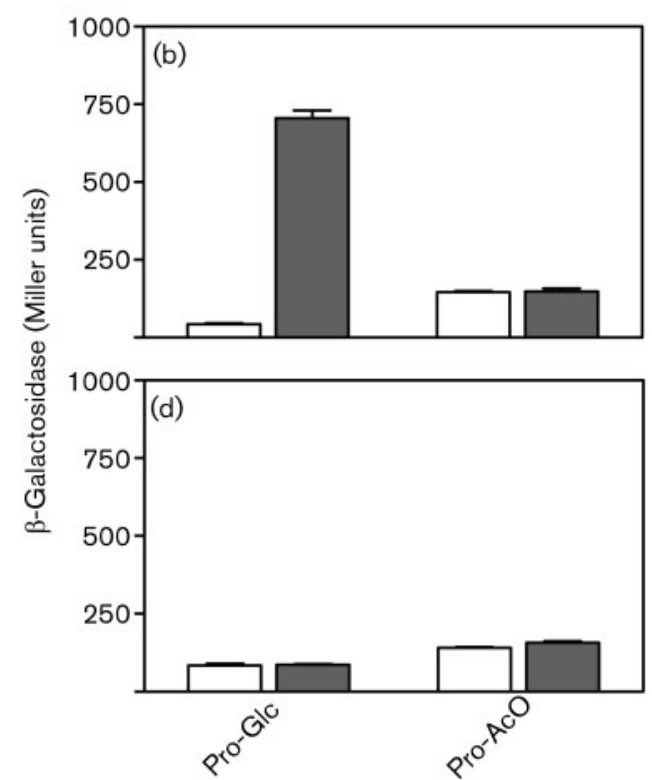

(c)

AAAAACCATTAGTAATAAT

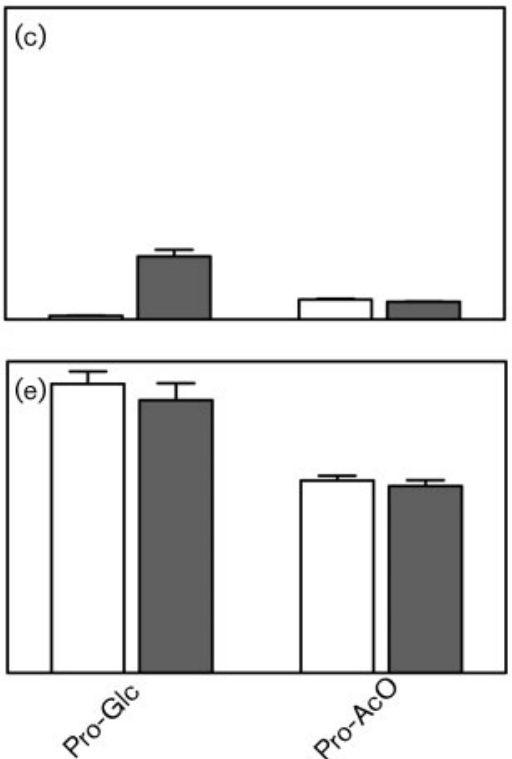

Fig. 5. Participation of the UAS elements in UGA4 regulation by carbon source. (a) Scheme of the fusion genes used. (b-e) $\beta$ Galactosidase activity was determined in extracts from wild-type (23344c strain) cells carrying the UGA4::lacZ (b), $U A S_{G A T A} \Delta$ : : lacZ (c), UASA : : lacZ (d) or UAS ${ }_{G A B A} m u t$ : : lacZ (e) fusion genes. Cells grown in minimal medium containing proline as the nitrogen source and glucose or acetate as the carbon source were collected and transferred to the same fresh media containing (filled bars) or not (open bars) $0.1 \mathrm{mM} \mathrm{GABA}$. After a $2 \mathrm{~h}$ incubation samples were taken and $\beta$-galactosidase activity was measured. Results are shown as mean \pm SD of duplicates within a representative assay. The deviation of these values from the mean was less than $15 \%$.

of Gln3 expression and it probably responds to changes in Gln3 localization too. However, there is another pathway participating in the regulation of UGA4 by carbon source since in the absence of the four GATA factors or in the absence of the $\mathrm{UAS}_{\mathrm{GATA}}$ element, UGA4 still responds to the carbon source. Our results also show that the $\mathrm{UAS}_{\mathrm{GABA}}$ element is the target of factors acting in response to the quality of the carbon source since expression driven by the promoter with a mutated $\mathrm{UAS}_{\mathrm{GABA}}$ becomes independent of the carbon source. Moreover, results using this mutated promoter clearly show that there is a negative factor acting on $\mathrm{UAS}_{\mathrm{GABA}}$ and its activity depends on the carbon source. It is known that, to be effective, $\mathrm{UAS}_{\mathrm{GABA}}$ requires two positive-acting proteins, namely pathway-specific Uga3p and pleiotropic Uga35p (Dal81p/DurLp) (Talibi et al., 1995). Thus two possibilities may arise in the light of this new evidence. The first one is that Uga3 and/or Uga35 may have a negative influence on UGA4 transcription. Our results argue against a direct negative function of these factors since UGA4 expression levels detected in cells deficient in Uga3 or Uga35, although not reproducible, were always lower than those in wild-type cells. The second possibility would be the action, not yet described, of another transcription factor on $\mathrm{UAS}_{\mathrm{GABA}}$. Even though the participation of Leu3 in UGA4 transcription has not been reported, its consensus site for the binding to DNA overlaps the $\mathrm{UAS}_{\mathrm{GABA}}$ element, suggesting that this factor may be the negative factor operating in UGA4 regulation. Moreover, Boer et al. (2005) recently reported the impact of the LEU3 deletion on transcriptional regulation of nitrogen metabolism in cells under different growth conditions; they found an increase in UGA4 and UGA3 transcription. The results presented in this work and preliminary results recently obtained in our laboratory together with data from Boer et al. (2005) support our present hypothesis which is that the interaction of Uga3 and Uga35 with others factors (protein or effector molecules) results in the formation of a repressor complex 
that acts on $\mathrm{UAS}_{\mathrm{GABA}}$ in a way that depends on the carbon source.

\section{ACKNOWLEDGEMENTS}

This work was supported by the research grant from the University of Buenos Aires (UBA) UBACYT X014 (2004-2007). C. L. is a student fellow of the UBA. S. B. C. is a post-graduate fellow of the Argentine National Research Council (CONICET). M. B. M. and S. C. G. hold the post of Associate Researchers at the same Council.

\section{REFERENCES}

Andre, B., Hein, C., Grenson, M. \& Jauniaux, J. C. (1993). Cloning and expression of the UGA4 gene coding for the inducible GABA-specific transport protein of Saccharomyces cerevisiae. Mol Gen Genet 237, $17-25$.

Andre, B., Talibi, D., Soussi Boudekou, S., Hein, C., Vissers, S. \& Coornaert, D. (1995). Two mutually exclusive regulatory systems inhibit UASGATA, a cluster of $5^{\prime}$-GAT(A/T)A-3' upstream from the UGA4 gene of Saccharomyces cerevisiae. Nucleic Acids Res 23, 558-564.

Beck, T. \& Hall, M. N. (1999). The TOR signalling pathway controls nuclear localization of nutrient-regulated transcription factors. Nature 402, 689-692.

Bermudez Moretti, M., Correa Garcia, S., Ramos, E. \& Batlle, A. (1996). $\delta$-Aminolevulinic acid uptake is mediated by the $\gamma$ aminobutyric acid-specific permease UGA4. Cell Mol Biol (Noisy-legrand) 42, 519-523.

Bertram, P. G., Choi, J. H., Carvalho, J., Chan, T. F., Ai, W. \& Zheng, X. F. (2002). Convergence of TOR-nitrogen and Snf1-glucose signaling pathways onto Gln3. Mol Cell Biol 22, 1246-1252.

Boer, V. M., Daran, J. M., Almering, M. J., de Winde, J. H. \& Pronk, J. T. (2005). Contribution of the Saccharomyces cerevisiae transcriptional regulator Leu3p to physiology and gene expression in nitrogen- and carbon-limited chemostat cultures. FEMS Yeast Res 5, 885-897.

Bricmont, P. A., Daugherty, J. R. \& Cooper, T. G. (1991). The DAL81 gene product is required for induced expression of two differently regulated nitrogen catabolic genes in Saccharomyces cerevisiae. Mol Cell Biol 11, 1161-1166.

Coffman, J. A., Rai, R., Loprete, D. M., Cunningham, T., Svetlov, V. \& Cooper, T. G. (1997). Cross regulation of four GATA factors that control nitrogen catabolic gene expression in Saccharomyces cerevisiae. J Bacteriol 179, 3416-3429.

Crespo, J. L., Powers, T., Fowler, B. \& Hall, M. N. (2002). The TORcontrolled transcription activators GLN3, RTG1, and RTG3 are regulated in response to intracellular levels of glutamine. Proc Natl Acad Sci U S A 99, 6784-6789.

Cunningham, T. S., Dorrington, R. A. \& Cooper, T. G. (1994). The UGA4 UASNTR site required for GLN3-dependent transcriptional activation also mediates DAL80-responsive regulation and DAL80 protein binding in Saccharomyces cerevisiae. J Bacteriol 176, 4718-4725.

Gietz, R. D. \& Woods, R. A. (2002). Transformation of yeast by lithium acetate/single-stranded carrier DNA/polyethylene glycol method. Methods Enzymol 350, 87-96.
Kelley, C., Blumberg, H., Zon, L. I. \& Evans, T. (1993). GATA-4 is a novel transcription factor expressed in endocardium of the developing heart. Development 118, 817-827.

Kulkarni, A. A., Abul-Hamd, A. T., Rai, R., El Berry, H. \& Cooper, T. G. (2001). Gln3p nuclear localization and interaction with Ure2p in Saccharomyces cerevisiae. J Biol Chem 276, 32136-32144.

Kulkarni, A., Buford, T. D., Rai, R. \& Cooper, T. G. (2006). Differing responses of Gat1 and Gln3 phosphorylation and localization to rapamycin and methionine sulfoximine treatment in Saccharomyces cerevisiae. FEMS Yeast Res 6, 218-229.

Kuruvilla, F. G., Shamji, A. F. \& Schreiber, S. L. (2001). Carbon- and nitrogen-quality signaling to translation are mediated by distinct GATA-type transcription factors. Proc Natl Acad Sci U S A 98, 7283-7288.

Kuruvilla, F. G., Shamji, A. F., Sternson, S. M., Hergenrother, P. J. \& Schreiber, S. L. (2002). Dissecting glucose signalling with diversityoriented synthesis and small-molecule microarrays. Nature 416, 653-657.

Laverriere, A. C., MacNeill, C., Mueller, C., Poelmann, R. E., Burch, J. B. \& Evans, T. (1994). GATA-4/5/6, a subfamily of three transcription factors transcribed in developing heart and gut. $J$ Biol Chem 269, 23177-23184.

Miller, J. H. (1972). Experiments in Molecular Genetics. Cold Spring Harbor, NY: Cold Spring Harbor Laboratory.

Myers, A. M., Tzagoloff, A., Kinney, D. M. \& Lusty, C. J. (1986). Yeast shuttle and integrative vectors with multiple cloning sites suitable for construction of lacZ fusions. Gene 45, 299-310.

Sambrook, J., Fritsch, E. F. \& Maniatis, T. (1997). Molecular Cloning: a Laboratory Manual. Cold Spring Harbor, NY: Cold Spring Harbor Laboratory.

Shamji, A. F., Kuruvilla, F. G. \& Schreiber, S. L. (2000). Partitioning the transcriptional program induced by rapamycin among the effectors of the Tor proteins. Curr Biol 10, 1574-1581.

Soussi-Boudekou, S., Vissers, S., Urrestarazu, A., Jauniaux, J. C. \& Andre, B. (1997). Gzf3p, a fourth GATA factor involved in nitrogenregulated transcription in Saccharomyces cerevisiae. Mol Microbiol 23, 1157-1168.

Strachan, T. \& Read, A. P. (1999). PCR, DNA sequencing and in vitro mutagenesis. In Human Molecular Genetics 2. London: Garland Science.

Talibi, D., Grenson, M. \& Andre, B. (1995). Cis- and trans-acting elements determining induction of the genes of the gammaaminobutyrate (GABA) utilization pathway in Saccharomyces cerevisiae. Nucleic Acids Res 23, 550-557.

Tate, J. J., Rai, R. \& Cooper, T. G. (2005). Methionine sulfoximine treatment and carbon starvation elicit Snf1-independent phosphorylation of the transcription activator Gln3 in Saccharomyces cerevisiae. J Biol Chem 280, 27195-27204.

Tate, J. J., Rai, R. \& Cooper, T. G. (2006). Ammonia-specific regulation of Gln3 localization in Saccharomyces cerevisiae by protein kinase Npr1. J Biol Chem 281, 28460-28469.

Weiss, M. J. \& Orkin, S. H. (1995). GATA transcription factors: key regulators of hematopoiesis. Exp Hematol 23, 99-107.

Edited by: D. Burke 